\title{
Probing Microstructure-Dependent Ionic Conductivity and Stability of Garnet Solid Electrolytes through In Situ TEM with Operando Impedance Spectroscopy
}

\author{
Hongkui Zheng ${ }^{1}$ and Kai $\mathrm{He}^{2}$ \\ ${ }^{1}$ Clemson University, United States, ${ }^{2}$ Clemson University, Clemson, South Carolina, United States
}

All solid-state batteries (ASSBs) have attracted extensive attention as a promising candidate for the large-scale energy storage alternative to the conventional lithium-ion batteries (LIBs). By replacing the flammable organic liquid electrolytes with the stable solid-state electrolytes, the inevitable safety issue of LIBs can be substantially mitigated, which may enable the use of alkaline metals as viable anode materials of ASSBs, leading to even higher energy density and packing density than the current LIB technology. Among various ceramic solid-state electrolytes, garnet-type $\mathrm{Li}_{7} \mathrm{La}_{3} \mathrm{Zr}_{2} \mathrm{O}_{12}$ (LLZO) has been extensively studied due to its high room-temperature Li-ion conductivity (up to $\sim 1 \mathrm{mS} / \mathrm{cm}$ ), large electrochemical window, and prominent stability against Li metal [1]. The inherent high shear modulus of ceramic LLZO electrolytes can effectively inhibit the growth of harmful Li dendrites [2]. However, critical challenges still exist in mechanistic understanding of the microstructure-dependent ionic conductivity and stability of the solid electrolyte and across the electrode-electrolyte interfaces.

Transmission electron microscopy (TEM) is an indispensable approach for the microstructural characterization. Recent advancements of in situ TEM techniques have enabled new capabilities to study dynamic reactions and electrochemical processes in battery systems [3]. Our recent technical development has overcome the barriers for characterizing Li metal and its vulnerable compounds susceptible to contamination and damage. For example, we have demonstrated the air-free cryogenic-FIB-TEM approach to preserve the pristine Li-containing materials from side reactions and electron radiation damage [4, 5]. Here, we report the new progress that allows for the operando electrochemical impedance spectroscopy (EIS) measurement of solid electrolytes during in situ TEM observation. Figure 1 shows the high-resolution TEM (HRTEM) image and X-ray diffraction (XRD) pattern of as-synthesized LLZO, confirming its high purity and high crystallinity. Figure 2 shows the entire experimental workflow kept air-free conditions. We first use focused ion beam (FIB) to prepare a thin lamella via a lift-out process and then transfer it onto a MEMS-chip to make an EIS testing structure sandwiched by two W blocking electrodes (Figure 2a). This MEMS device can air-free transfer to TEM for chemical mapping and microstructural analysis (Figure 2b, c). Simultaneously, we can also perform DC/AC impedance measurements directly on this individual LLZO sample. Our initial control experiments show that the DC resistance of LLZO has increased $\sim 6$ times after exposure to air (Figure 2d). The EIS Nyquist plots indicate that the AC impedance may slightly increase under electron beam illumination (Figure 2e). These results show consistent implications that the alteration of the LLZO microstructure (grain boundaries, pores, and other local defects) has a direct impact on its ionic conductivity. However, the detailed microstructureconductivity correlation and the governing mechanism need to be clarified by further investigation. Our methodology combining in situ TEM and operando EIS has demonstrated its feasibility and effectiveness in a prototype LLZO device, which has paved the way for unlocking the hidden microstructure dependence on ionic conductivity and stability for general solid-state electrolyte and electrolyte-electrode interfacial systems [6]. 

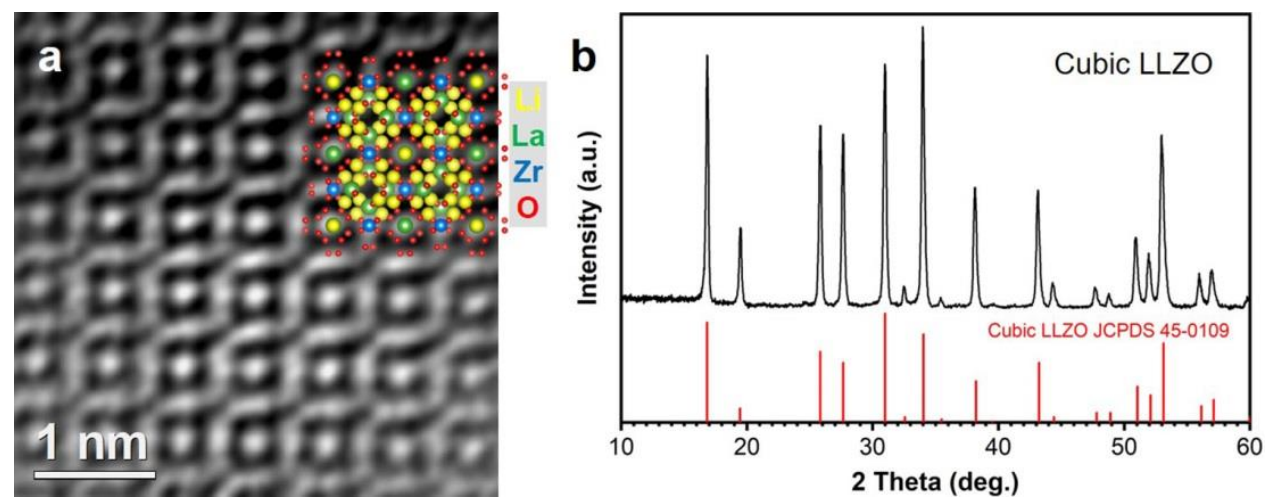

Figure 1. Figure 1. (a) HRTEM image with the overlaid unit-cell model, and (b) XRD pattern of as-synthesized LLZO solid electrolyte.
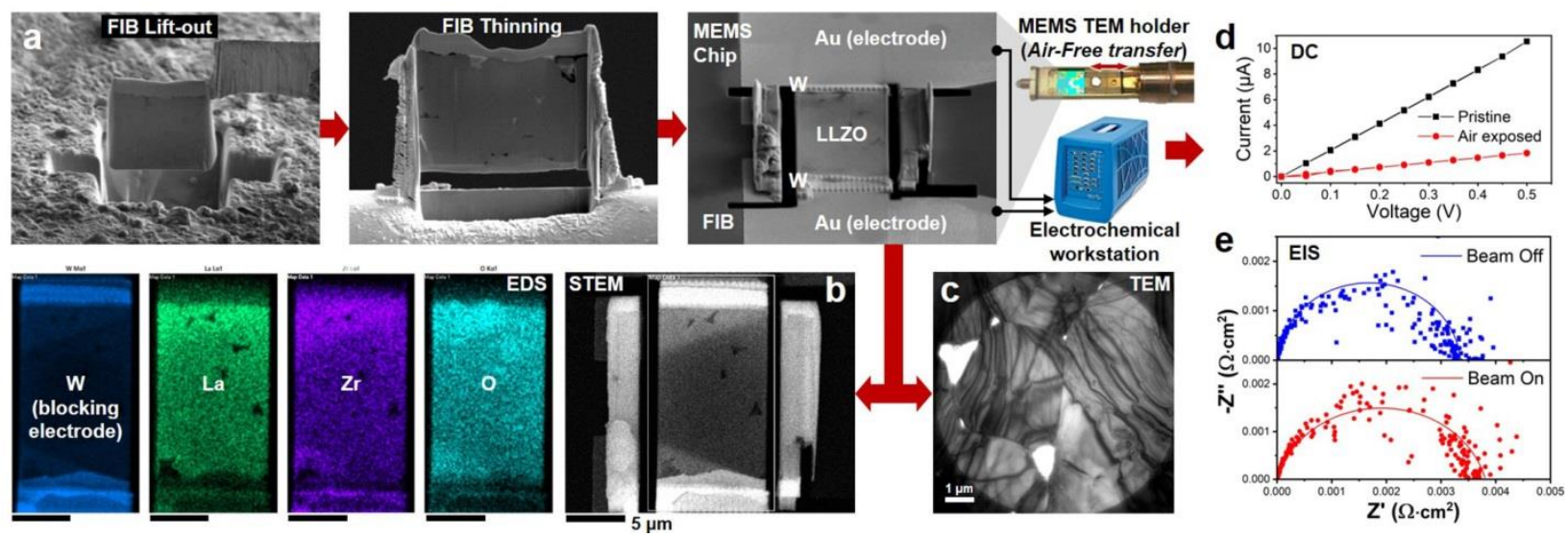

Figure 2. Figure 2. Prototype instrumentation and experimental workflow to link (a) FIB preparation with (b) STEM-EDS chemical mapping and (c) TEM microstructural analysis, and further correlate to (d) DC resistant and (e) AC EIS measurements. The entire procedure is kept air-free to protect sample from damage, contamination and side reactions.

\section{References}

[1] V. Thangadurai et al., Chem. Soc. Rev. 43, 4714 (2014).

[2] H. Liu et al., ACS Energy Lett. 5, 833 (2020).

[3] J. Cui et al., Adv. Mater. 33, 2000699 (2021).

[4] H. Zheng et al., Microsc. Microanal. 26, 2784 (2020).

[5] J. Cui et al., Microsc. Microanal. 26, 1828 (2020).

[6] The authors acknowledge the use of Electron Microscopy Facility at Clemson University. 